\title{
Effective critical behavior of the two-dimensional Ising spin glass with bimodal interactions
}

\author{
Helmut G. Katzgraber, ${ }^{1}$ L. W. Lee, ${ }^{2}$ and I. A. Campbell ${ }^{3}$ \\ ${ }^{1}$ Theoretische Physik, ETH Zürich, CH-8093 Zürich, Switzerland \\ ${ }^{2}$ Department of Physics, University of California, Santa Cruz, California 95064, USA \\ ${ }^{3}$ Laboratoire des Colloïdes, Verres et Nanomateriaux, \\ Université Montpellier II, 34095 Montpellier, France
}

(Dated: September 14, 2018)

\begin{abstract}
Monte Carlo data of the two-dimensional Ising spin glass with bimodal interactions are presented with the aim of understanding the low-temperature physics of the model. An analysis of the specific heat, spin-glass susceptibility, finite-size correlation length, and the Binder ratio is performed to try to verify a recent proposal in which for large system sizes and finite but low temperatures the effective critical exponents are identical to the critical exponents of the two-dimensional Ising spin glass with Gaussian interactions. Our results show that with present system sizes the recently proposed scenario in which the two-dimensional Ising spin glass with bimodally distributed interactions is in the same universality class as the model with Gaussian-distributed disorder at low but finite temperatures cannot be reliably proven.
\end{abstract}

PACS numbers: $75.50 . \mathrm{Lk}, 75.40 . \mathrm{Mg}, 05.50 .+\mathrm{q}$

\section{INTRODUCTION}

The two-dimensional Ising spin glass $\frac{1}{1}$ has been the subject of numerous numerical studies $2,3,4,5,6$ ever since its introduction by Edwards and Anderson in $1975 \stackrel{\underline{7}}{\mathrm{D}}$ Despite the fact that this canonical model only orders at zero temperature, its popularity can be ascribed mainly to its ease of implementation and simplicity. Yet it has proven to be extremely difficult to establish if the model orders at zero or finite temperature and what the exact values of the critical exponents are. Today it is well recognized that the model only orders at zero temperature $\underline{\underline{8}}$ In addition, the critical exponents at $T=0$ are known to good precision. $\frac{8}{}$

When the interactions between the spins are drawn from a Gaussian distribution with zero mean, the system has a unique ground state (up to a global symmetry). This in turn implies that the critical exponent of the correlation function has to be zero, i.e., $\eta=2-d=0$, where $d=2$ is the space dimension. In addition, extensive zero-temperature domain-wall computations have established that the domain-wall stiffness exponent is $\theta=-0.282(2) \stackrel{8.9}{.}$ Because at a zero-temperature transition the critical exponent of the correlation length $\nu$ is related to $\theta$ via $\nu=-1 / \theta$, it follows that $\nu=3.54(2)$.

The ground state of the model with bimodally distributed random bonds, on the other hand, is strongly degenerate ${ }^{5,10}$ with a finite residual entropy at $T=0$. Domain-wall stiffness measurements at zero temperature show an exponent $\theta=0^{\underline{8}}$ (exponential scaling) although with significant corrections to scaling up to linear system sizes $L \approx 100$ under periodic/free boundary conditions. Direct measurements of the spin-spin correlation function $G(r)$ at $T=0$ indicate a critical exponent $\eta \approx 0.14{ }^{11}$ An extrapolation from finite temperatures $\underline{\underline{\underline{6}}}$ yielded to high precision $\eta=0.138(5)$. Other estimates of $\eta$ have given positive values in the range $0.14-0.40,2,3,10,12,13,14$
Judging from their zero-temperature (critical) properties the two versions of the model thus are in two different universality classes (see Table III). However, for low but finite temperatures the situation is less clear-cut. For the model with bimodal disorder, when a sample is in a ground state, turning over one individual spin either leaves the total energy of the sample unchanged or increases it by $4 J$ or $8 J$, where $J$ is the characteristic energy of the model (see below). This suggests immediately that there is an energy gap of $A J$ between the ground state and the first excited state, with $A=4$. It has been argued however that the true elementary excitations are not single spins but more complex objects, leading to an effective gap of $\sim 2 J \underline{2,4,5,15}$ Because of the gapped excitation spectrum we expect a crossover temperature $T^{*}(L)$, which separates a critical behavior, in accordance with the aforementioned zerotemperature critical point and a critical behavior which would resemble a model with continuous interactions. If the ground-state degeneracy is given by $N_{0}(L)$ and the excited states have a degeneracy $N_{1}(L), N_{2}(L), \ldots$, for $N_{0}(L) \gg N_{1}(L) \exp (-4 J / T)$ the system will spend almost all of its time in the ground state and equilibrium properties will be essentially those of the ground-state manifold, whereas for $N_{1}(L) \exp (-4 J / T) \gg N_{0}(L)$ the system will stay in the quantized series of excited states and the properties of the system can be expected to resemble those of a system with no gap in the energy spectrum. Note that this argument is slightly oversimplified as the effects of higher excited states on $T^{*}(L)$ are not taken into account, yet we expect their contributions to be small $\frac{16}{}$ One obtains to lowest order $T^{*}(L) \sim 4 J / \ln \left[N_{1}(L) / N_{0}(L)\right]$. The ratio $N_{1}(L) / N_{0}(L)$ with a gap $4 J$ has been estimated in Refs. 5 and 16 and shows that $T^{*}(L)$ drops gradually as $L$ increases. Our data presented below confirm this behavior.

It has been strongly argued $\frac{17}{17}$ that in the limit $T>$ 
$T^{*}(L)$ and with $L \rightarrow \infty$ [meaning $T^{*}(L)$ tending to zero but never reaching zero] the model with bimodal disorder has effective critical exponents identical to the critical exponents of the model with Gaussian-distributed disorder, so that the two models can be considered as being in the same universality class except for the singular behavior of the model with bimodally distributed disorder at $T=0$.

We present the results of Monte Carlo simulations of the two-dimensional Ising spin glass with bimodally distributed disorder on system sizes larger than those used in Refs. 5 and 17. Our results show that with current algorithms and computer power the data do not provide definitive limiting values for the critical exponents of the model, although power-law scaling seems plausible for finite but nonzero temperatures. Therefore the claim that the aforementioned model is in the same universality class at finite but nonzero temperatures as the model with Gaussian, gap 1/4, or diluted interactions 17 remains to be proven.

The paper is structured as follows: In Sec. II we introduce the model, numerical method, and observables, and discuss different finite-size scaling relations. In Sec. III we summarize previous results on the two-dimensional Ising spin glass with bimodally distributed disorder. Results on the different critical exponents are presented in Sec. IV] and a finite-size scaling analysis of the data is presented in Sec. V.

\section{MODEL, OBSERVABLES, AND FINITE-SIZE SCALING RELATIONS}

The Hamiltonian of the two-dimensional Ising spin glass is given by

$$
\mathcal{H}=-\sum_{\langle i, j\rangle} J_{i j} S_{i} S_{j}
$$

$S_{i}= \pm 1$ represent Ising spins and the sum is over nearest neighbors on a square lattice with periodic boundary conditions. The interactions $J_{i j} \in\{ \pm J\}$ (here $J=1$ ) are bimodally distributed. For the Monte Carlo simulations we use a combination of single-spin flips, exchange Monte Carlo updates,$\frac{18,19}{19}$ and rejection-free cluster moves ${ }^{4}$ to speed up equilibration. Equilibration of the method is tested by performing a logarithmic data binning of all observables, and we require that the last three bins agree within error bars and are independent of the number of Monte Carlo sweeps $N_{\text {sweep }}$. The parameters of the simulation are listed in Table [.

The second-moment finite-size correlation length $\frac{20,21,22,23,24,25}{2} \xi_{L}$ is given by

$$
\xi_{L}=\frac{1}{2 \sin \left(\left|\mathbf{k}_{\min }\right| / 2\right)}\left[\frac{\chi_{\mathrm{SG}}(0)}{\chi_{\mathrm{SG}}\left(\mathbf{k}_{\min }\right)}-1\right]^{1 / 2},
$$

where $\mathbf{k}_{\min }=(2 \pi / L, 0)$ is the smallest nonzero wave vector, and $\chi_{\mathrm{SG}}(\mathbf{k})$ is the wave-vector-dependent spin-glass
TABLE I: Parameters of the simulations. $N_{\text {samp }}$ represents the number of disorder realizations computed; $N_{\text {sweep }}$ is the total number of Monte Carlo sweeps of the $2 N_{T}$ replicas for a single sample. $N_{T}$ is the number of temperatures in the exchange Monte Carlo method and $T_{\min }$ represents the lowest temperature simulated. (For $L=128$ no data for the specific heat has been generated.)

\begin{tabular}{crrrr}
\hline \hline$L$ & $N_{\text {samp }}$ & $N_{\text {sweep }}$ & $T_{\min }$ & $N_{\mathrm{T}}$ \\
\hline 32 & 5000 & $2.0 \times 10^{6}$ & 0.050 & 20 \\
48 & 1000 & $2.0 \times 10^{6}$ & 0.050 & 20 \\
64 & 500 & $4.2 \times 10^{6}$ & 0.200 & 39 \\
96 & 609 & $6.5 \times 10^{6}$ & 0.200 & 63 \\
128 & 420 & $2.0 \times 10^{6}$ & 0.396 & 50 \\
\hline \hline
\end{tabular}

susceptibility,

$$
\chi_{\mathrm{SG}}(\mathbf{k})=\frac{1}{N} \sum_{i, j}\left[\left\langle S_{i} S_{j}\right\rangle^{2}\right]_{\mathrm{av}} e^{i \mathbf{k} \cdot\left(\mathbf{R}_{i}-\mathbf{R}_{j}\right)} .
$$

In the previous equation $[\cdots]_{\mathrm{av}}$ represents a disorder average and $\langle\cdots\rangle$ a thermal average. The finite-size correlation length is expected to scale as

$$
\xi_{L} \sim\left(T-T_{\mathrm{c}}\right)^{-\nu}
$$

where $\nu$ is the critical exponent for the correlation length. This scaling behavior is expected to also be valid for zerotemperature transitions when the ground state is not degenerate. Since in this work we want to study the thermodynamic limit at finite but nonzero temperatures, we postulate that the scaling ansatz in Eq. (4) also holds for the model with a bimodal disorder distribution when $T>0$.

The standard spin-glass susceptibility $\chi_{\mathrm{SG}}=\chi_{\mathrm{SG}}(\mathbf{k}=$ 0 ) can also be defined via $\chi_{\mathrm{SG}}=N\left[\left\langle q^{2}\right\rangle\right]_{\mathrm{av}}$, where

$$
q=\frac{1}{N} \sum_{i=1}^{N} S_{i}^{a} S_{i}^{b} .
$$

In Eq. (5) $\left\{S_{i}^{a}\right\}$ and $\left\{S_{i}^{b}\right\}$ are two copies of the system with the same disorder. According to finite-size scaling we expect that

$$
\chi_{\mathrm{SG}} \sim\left(T-T_{\mathrm{c}}\right)^{-\gamma},
$$

and at criticality $\chi_{\mathrm{SG}}\left(T=T_{\mathrm{c}}\right) \sim L^{2-\eta}$, where $\eta$ is the anomalous dimension exponent of the correlation function $G(r)$,

$$
G(r, T)=\left[\left\langle S_{i} S_{i+r}\right\rangle^{2}\right]_{\mathrm{av}}=\frac{1}{r^{d-2+\eta}} e^{-r / \xi(T)} .
$$

In addition, we study the dimensionless Binder ratio $\underline{26}$ defined via

$$
g=\frac{1}{2}\left[3-\frac{\left[\left\langle q^{4}\right\rangle\right]_{\mathrm{av}}}{\left[\left\langle q^{2}\right\rangle\right]_{\mathrm{av}}^{2}}\right] .
$$

In Sec. $\mathrm{V}$ we plot the Binder ratio as a function of the correlation length divided by the system size. The method 
has the advantage that if data for different disorder distributions lie on the same universal curve, the systems are in the same universality class. ${ }^{27}$ Finally, we also compute the specific heat of the system, ${ }^{28}$

$$
C_{\mathrm{V}}=\frac{1}{T^{2}}\left[\left\langle\mathcal{H}^{2}\right\rangle-\langle\mathcal{H}\rangle^{2}\right]_{\mathrm{av}},
$$

which is expected to scale as

$$
C_{\mathrm{V}} \sim\left(T-T_{\mathrm{c}}\right)^{-\alpha}
$$

For zero-temperature transitions the critical contribution of the specific heat can also be written as $C_{\mathrm{V}}(T) \sim T^{d \nu}$ using zero-transition-temperature scaling relations, ${ }^{29,30}$

If two systems are in the same universality class they share identical values of the critical exponents, as well as the values of different observables at criticality [e.g., $\left.g\left(T_{\mathrm{c}}\right)\right]^{31}$ Assuming the power-law behaviors for the different observables [Eqs. (4), (6), and (10)] at low but nonzero temperature, we study the values of the effective critical exponents.

\section{SUMMARY OF PREVIOUS RESULTS}

The critical properties of the two-dimensional Ising spin glass with Gaussian-distributed interactions are firmly established from zero-temperature simulations. Because the ground state is not degenerate, the correlation function $G(r, T)=1$ for all $r$ at $T=T_{\mathrm{c}}=0$. Therefore, by definition, $\eta=0, \xi(T=0)=\infty$, and for the Binder ratio at zero temperature $g(T=0)=1$. It is now well established from domain-wall measurements at $T=0$, confirmed by size-dependent ground-state energy measurements 29 that $\theta=-0.282(2)$, hence the thermal exponent $\nu$ (whose value is not fixed by the unique ground-state condition) is $\nu \equiv-1 / \theta=3.54(2)$. Measurements of $\nu$ at finite temperatures via Monte Carlo simulations give consistent estimates, $\stackrel{25}{ }$ yet only if large enough system sizes are simulated. The different expected critical exponents at zero temperature are summarized in Table II]

For the two-dimensional Ising spin glass with bimodaldistributed interactions the situation is, however, much more complicated because of the highly degenerate ground state, as well as the quantized energy spectrum. As noted for instance in Ref. [5, the naïve prediction for the low-temperature limit specific heat for the twodimensional system of size $L$ and gap $A=4$ is

$$
C_{\mathrm{V}}(T)=\frac{16}{(T L)^{2}} \frac{N_{1}(L)}{N_{0}(L)} e^{-4 J / T}
$$

A priori this low-temperature exponential finite-size behavior should always hold for $T \ll T^{*}(L)$. Surprisingly, there has been a longstanding controversy concerning the low-temperature behavior of the specific heat in the thermodynamic limit. An exponential scaling of the
TABLE II: Critical exponents for both disorder distributions at zero temperature. For the bimodal disorder distribution, exponential scaling is expected, i.e., $\nu=\infty$ (see the main text for details). The critical exponent $\eta$ in the bimodal case follows from Refs. 12, 13, 2, 3, 10, and 14 and are estimated by extrapolating finite-temperature data to $T=0$. In the Gaussian case the estimate for $\nu$ is from Ref. 8. The remaining critical exponents can be computed from the zero-temperature scaling relations $\alpha=-d \nu$ and $\gamma=\nu(d-\eta)$, where $d=2$ is the space dimension.

\begin{tabular}{lll}
\hline \hline Disorder & $\nu$ & $\eta$ \\
\hline Gaussian & $3.54(2)$ & 0 \\
Bimodal & $\infty$ & $0.14-0.40$ \\
\hline \hline
\end{tabular}

free energy, and thus correspondingly of all thermodynamic quantities, has been first proposed by Wang and Swendsen ${ }^{2}$ They surmised that

$$
C_{\mathrm{V}} \sim \frac{1}{T^{P}} e^{-A J / T}
$$

The numerical parameters $A=4$ and $P=2$ can be expected from the aforementioned arguments regarding the gap in the excitation spectrum. In addition, according to hyperscaling, the singular part of the free energy scales as $\xi^{-d}$ with $d=2$ and so, if $C_{\mathrm{V}}$ scales exponentially, we expect that the correlation length $\xi$ scales as

$$
\xi \sim e^{n J / T}
$$

with $n=A / 2$, as predicted first by Saul and Kardar $\underline{3}^{3}$

Wang and Swendsen ${ }^{2}$ calculated numerically the specific heat of the model. Surprisingly, they found $A=2$, thus suggesting a nontrivial scaling of the free energy. However, their measurements were restricted to small system sizes and to few disorder realizations, and their results indicated strong corrections to scaling. These conclusions stand in contrast to those from work by Saul and Kardar,$\underline{3}$ who argue that $A=4$. In addition, these authors also estimated $n=2$, a behavior which appeared to be confirmed independently in work by Houdayer ${ }^{4}$ who studied the finite-size scaling of the Binder ratio 26 as well as by Katzgraber et $a l \underline{\underline{6}}$ who were the first to study the finite-size scaling of the finite-size correlation length directly via Monte Carlo simulations. Note that an exponential scaling of the correlation length (for all $A$ ) implies that the effective critical exponent $\nu$ is infinite. The Wang and Swendsen ${ }^{2}$ value for $A(A=2)$ was strongly supported by the numerical work of Lukic et al. $\frac{5}{n}$ who computed the specific heat of the model for intermediate system sizes $(L \leq 50)$, using Pfaffian matrix algebra techniques. From their analysis they concluded that $A$ could be estimated very accurately and that $C_{\mathrm{V}}(T)$ tends to the functional form in Eq. (12) with $P=2$ and $A=2.02(3), 32$ in agreement with the results of Ref. 2 . Recently, a new scenario has been proposed in Ref. 17: While at zero temperature the model with bimodally distributed couplings still exhibits exponential scaling with 
$A=4$, at finite but low temperatures in the thermodynamic limit the two-dimensional Ising spin glass with bimodal couplings falls into the same universality class as the system with Gaussian-distributed disorder. For nonzero temperatures and in the thermodynamic limit the observables are claimed to display power-law singularities with the same critical exponents as the system with Gaussian disorder. In this work we compute effective critical exponents with systems larger than in Ref. 17 in the temperature range where power-law scaling is expected to occur 17 ( $0.2 \lesssim T \lesssim 0.5$ for $L \gtrsim 50)$ and show that if the observables can be interpreted to exhibit power-law scaling, the critical exponents for the system with bimodally distributed couplings cannot be estimated reliably $\underline{33}$ from the system sizes studied in Ref. 17 .

\section{RESULTS}

We present numerical data on various observables, comparing where appropriate the systems with bimodal and Gaussian disorder. We note that for the model with bimodal disorder in addition to the crossover temperature $T^{*}(L)$ due to the energy gap, there is a finitesize crossover temperature $T_{\xi}(L)$ fixed by the condition $L \sim \xi(T)$. Above $T_{\xi}(L)$, the observables are close to the thermodynamic limit values while below $T_{\xi}(L), \chi_{\mathrm{SG}}$ and $\xi_{L}$ tend to be size-limited and thus temperature independent. For all system sizes studied we find $T_{\xi}(L)>T^{*}(L)$, meaning that as $T$ is lowered the size limited condition on $\xi_{L}$ and $\chi_{\mathrm{SG}}$ sets in well before the effect of the gap. The specific heat is approximately size independent down to $T^{*}$, but is strongly affected by the gap: below $T^{*}(L)$ the specific heat drops exponentially with decreasing $T$.

We now test the hypothesis of Ref. 17 that the effective finite-temperature exponents $\eta_{\text {eff }}$ and $\nu_{\text {eff }}$ exist and are identical to the values with Gaussian disorder in $d=2$, which are $\eta=0$ and $\nu=3.54(2)$, respectively. These values imply that the exponent of the specific heat is $\alpha \approx-7.1(\alpha=-d \nu)$ and for the susceptibility exponent $\gamma \approx 7.1[\gamma=\nu(d-\eta)]$.

\section{A. Specific heat}

Figure 1 shows a log-log plot of the specific heat $C_{\mathrm{V}}(L, T)$ as a function of $T$. The numerical results are consistent with those of the analogous plot shown in Fig. 4 of Ref. 17, but the present data extend to $L=96$. The slope in the range $0.2 \lesssim T \lesssim 0.5$ is $-d \ln \left[C_{\mathrm{V}}(L, T)\right] / d \ln [T] \sim-4.21(2)$, i.e., $\alpha \approx-4.21$. We also perform a point-by-point differential of the data for all $T$ (using a second-order midpoint differentiation combined with a bootstrap analysis to estimate the error bars) and thus estimate the effective exponent $\alpha_{\text {eff }}(L, T)$ as a function of temperature. Figure 2 displays $\alpha_{\mathrm{eff}}(L, T)=-d \ln \left[C_{\mathrm{V}}(L, T)\right] / d \ln [T]$ as a function of $T . \alpha_{\text {eff }}(L, T)$ should tend to the thermodynamic

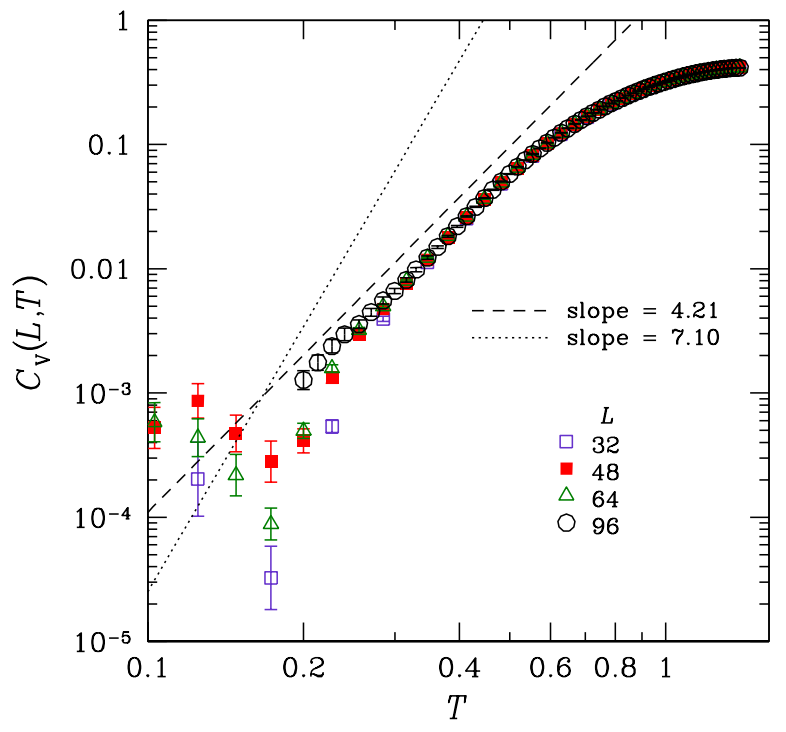

FIG. 1: (Color online) Log-log plot of the specific heat $C_{\mathrm{V}}$ vs temperature $T$ for several system sizes up to $L=96$. For $0.2 \lesssim T \lesssim 0.5$ the data seem to be approximately independent of system size and follow a power-law behavior, i.e., $C_{\mathrm{V}} \sim$ $T^{-\alpha}$ with $\alpha \approx-4.21(2)$ (the dashed line with slope 4.21 is a guide to the eye). For the system sizes studied, the data seem incompatible with a low-temperature, large-size-limiting effective exponent $\alpha$ equal to the Gaussian estimate for the critical exponent $\alpha \approx-7.1$ (dotted line with slope 7.1 ). The fluctuations at $T \lesssim 0.2$ can be ascribed to the exponential behavior at low enough temperatures.

critical exponent $\alpha_{\mathrm{eff}}$ in the limit $L \rightarrow \infty$ followed by $T \rightarrow 0$. An extrapolation of the data for $T>T^{*}(L)$, where $-d \ln \left[C_{\mathrm{V}}(L, T)\right] / d \ln [T]$ is independent of system size $L$, cannot be performed in a reliable way to test if the effective exponent agrees with the expected Gaussian value of $\alpha_{\text {eff }} \approx-7.1$.

For $0.2 \lesssim T \lesssim 0.5$ the data seem to saturate (see Fig. 2) although strong fluctuations are present. This "plateau region" resembles the behavior predicted by Fisch $\underline{16}$ who argues that there should be a rather broad region in temperature just above $T^{*}(L)$ for large (but not infinite) $L$, where $C_{\mathrm{V}} \sim T^{x}$ with $x=5.25(20)$. This is equivalent to a temperature-independent $\alpha_{\text {eff }}=-5.25$. Given the uncertainties in the estimate of the exponent, the agreement between the prediction by Fisch and the Monte Carlo data presented here is reasonably good. For the system sizes studied, which are larger than the ones studied in Ref. 17, the exponent $\alpha_{\text {eff }}$ in this large- $L$ low- $T$ region seems to be different from the Gaussian critical exponent $\alpha=-2 \nu \approx-7.1$. (In fact, in the Gaussian model the low-temperature specific heat is dominated by noncritical contributions ${ }^{-5}$ and the true critical behavior is not directly visible). Therefore, if both models share the same universality class, the system with bimodal disorder 


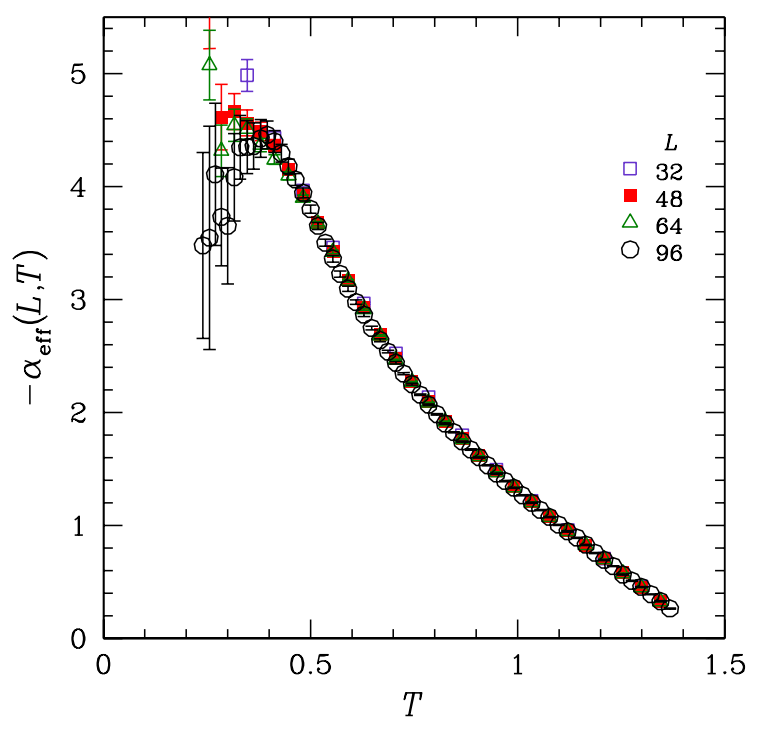

FIG. 2: (Color online) Effective exponent $\alpha_{\text {eff }}(L, T)=$ $-d \ln \left[C_{\mathrm{V}}(L, T)\right] / d \ln [T]$ as a function of temperature for different system sizes $L$. For the system sizes shown, the data cannot be extrapolated in a reliable way to $\alpha_{\text {eff }}=-7.1$, the value obtained for the model with Gaussian-disordered bonds. Therefore it is rather difficult to test if the effective critical exponents agree with the known Gaussian values.

displays huge corrections to scaling and thus simulations at considerably larger system sizes would be required to prove this beyond any reasonable doubt.

\section{B. Correlation length and susceptibility}

From the assumed power-law critical behavior of the spin-glass susceptibility one can define an effective exponent $\gamma_{\mathrm{eff}}(L, T)=-d \ln \left[\chi_{\mathrm{SG}}(L, T)\right] / d \ln [T]$ using point-by-point differentiation. Figure 3 shows $\gamma_{\text {eff }}(L, T)$ against $T$. The thermodynamic limit domain where the effective exponent is size independent can be seen clearly; for each $L$ the data peel off the thermodynamic limit line at the finite-size limited $T_{\xi}(L)$. However, even with data up to $L=128$ there is no reliable way to extrapolate to the critical value at infinite $L$ and $T$ tending to zero. A similar conclusion can be reached for $\nu_{\text {eff }}(L, T)=-d \ln [\xi(L, T)] / d \ln [T]$, Fig. 4. The error bars in Figs. 2, 3, and 4 have been calculated via a bootstrap estimate $\underline{\underline{34}}$

In Ref. 17 the Caracciolo finite-size scaling technique ${ }^{22,35}$ was used to extrapolate $\xi(L, T)$ and $\chi_{\mathrm{SG}}(L, T)$ data for the $\pm J$ model towards infinite size, in order to extract the exponent $\eta$ from the critical scaling relation $\chi_{\mathrm{SG}}(L, T) \sim \xi(L, T)^{2-\eta}$. The data are interpreted as showing that $\eta \sim 0.17$

This technique assumes that there are well-behaved

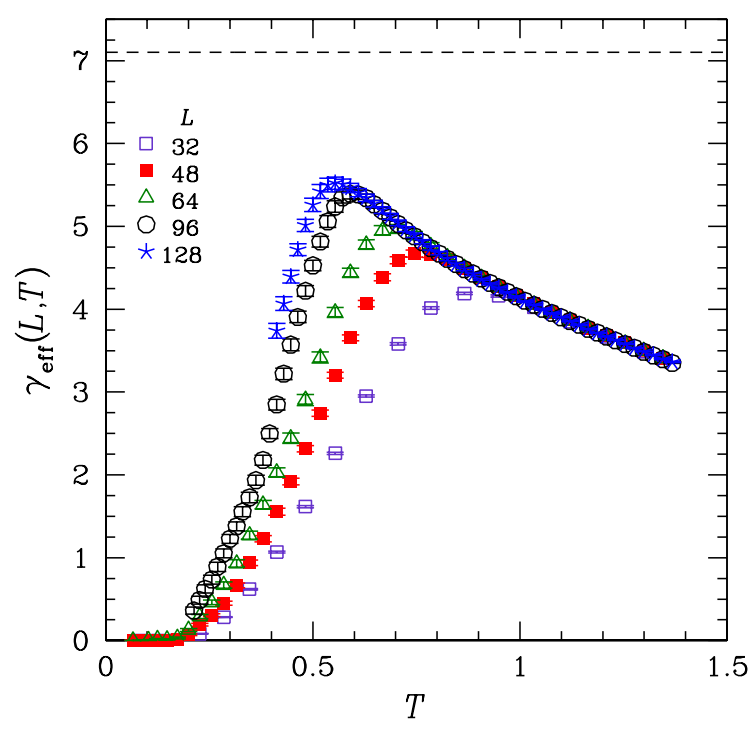

FIG. 3: (Color online) Effective exponent $\gamma_{\text {eff }}(L, T)=$ $-d \ln \left[\chi_{\mathrm{SG}}(L, T)\right] / d \ln [T]$ as a function of temperature for different system sizes $L$. An extrapolation to the lowtemperature regime is difficult with system sizes limited to $L \leq 128$. The horizontal dashed line corresponds to the expected Gaussian value $\gamma \approx 7.1$. An agreement or disagreement with $\gamma \approx 7.1$ cannot be ruled out.

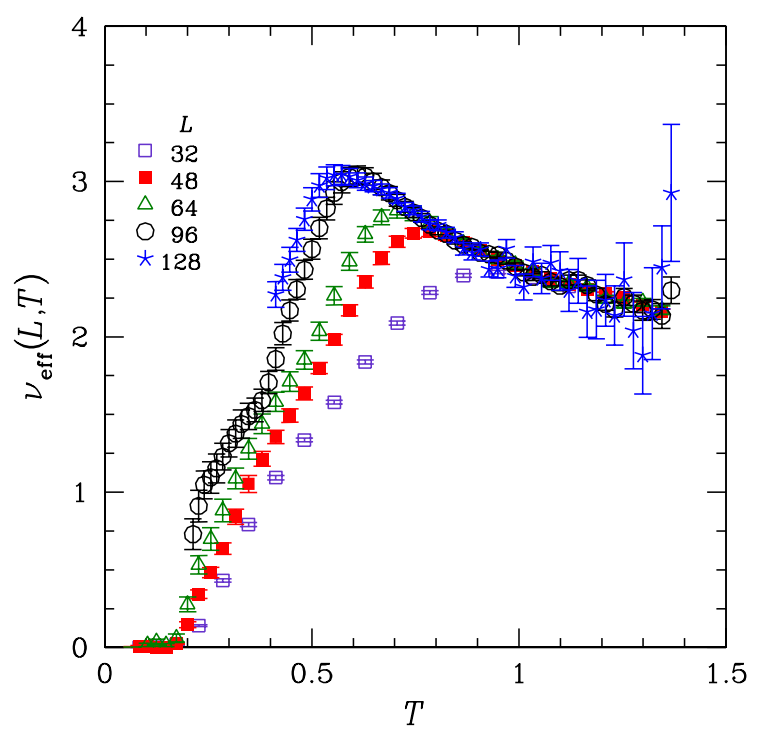

FIG. 4: (Color online) Effective exponent $\nu_{\text {eff }}(L, T)=$ $-d \ln [\xi(L, T)] / d \ln [T]$ as a function of temperature for different system sizes $L$. An extrapolation to the low-temperature regime is difficult with system sizes limited to $L \leq 128$. Any extrapolation to the low- $T$ behavior where $\nu \approx 3.54$ would be difficult to perform with the current data. 


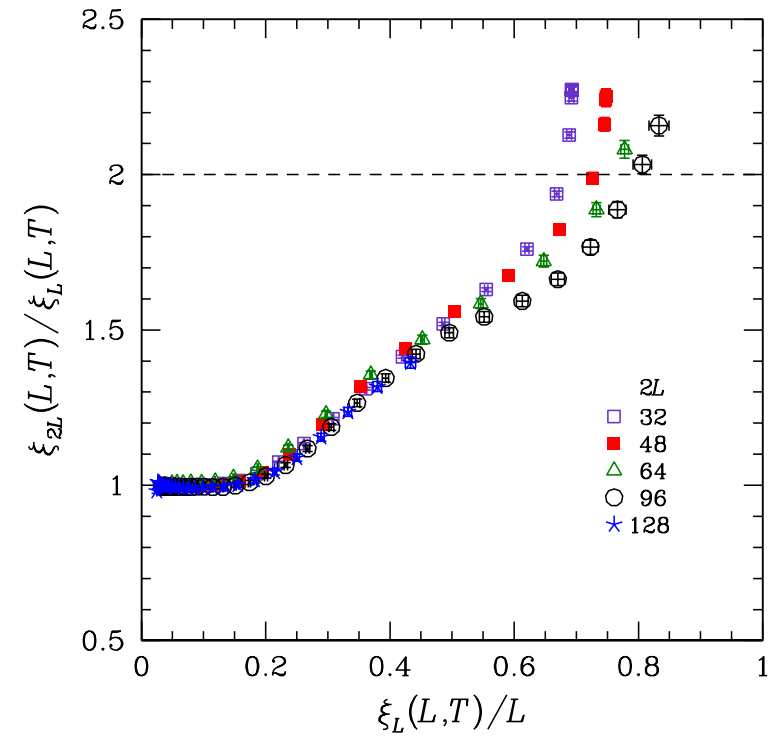

FIG. 5: (Color online) $\xi(2 L, T) / \xi(L, T)$ against $\xi(L, T) / L$. If corrections to scaling were small, all data should lie on an universal curve. This is a prerequisite to perform an extrapolation to the bulk regime. Clearly, the data do not scale well.

scaling functions for $\xi(L, T)$ and $\chi_{\mathrm{SG}}(L, T)$, such that, in particular, $\xi(2 L, T) / \xi(L, T)$ is a unique function of $\xi(L, T) / L$. Otherwise the Caracciolo scaling procedure cannot be applied as systematic errors would be introduced. Figure 5 shows data for $\xi(2 L, T) / \xi(L, T)$ vs $\xi(L, T) / L$ with $L$ between 16 and 48 . It can be seen that the finite-size corrections to scaling are strong because the curves for different $L$ do not superimpose, and also because the ratios $\xi(2 L, T) / \xi(L, T)$ increase beyond a value of 2 at low temperatures (in the absence of corrections 2 is the strict $T_{\mathrm{c}}$ limit of the ratio). This means that the Caracciolo procedure (or any similar protocol such as the one by $\mathrm{Kim}^{21}$ used by Katzgraber et al $l^{25}$ for the model with Gaussian disorder) has to be performed with considerable care for bimodally distributed disorder in two space dimensions. In Fig. [6 we show a log-log plot of the correlation length as a function of temperature together with an extrapolation using the methods of $\mathrm{Kim}^{21}$ as well as Palassini and Caraciolo. $\stackrel{22.35}{ }$ Both extrapolations agree very well. While the extrapolated data seem to follow a power-law behavior with $\nu \approx 3.45$, the extrapolation method is not reliable due to strong corrections to scaling (see Fig. 51). Until data on much larger system sizes become available it does not seem plausible to give a reliable account of the large- $L$, low- $T$ limiting functional behavior of $\chi_{\mathrm{SG}}(L, T)$ or $\xi(L, T)$.

Alternatively, with no extrapolation, one can define an effective exponent

$$
2-\eta_{\mathrm{eff}}(L, T)=-\frac{d \ln \left[\chi_{\mathrm{SG}}(L, T)\right]}{d \ln [\xi(L, T)]}
$$

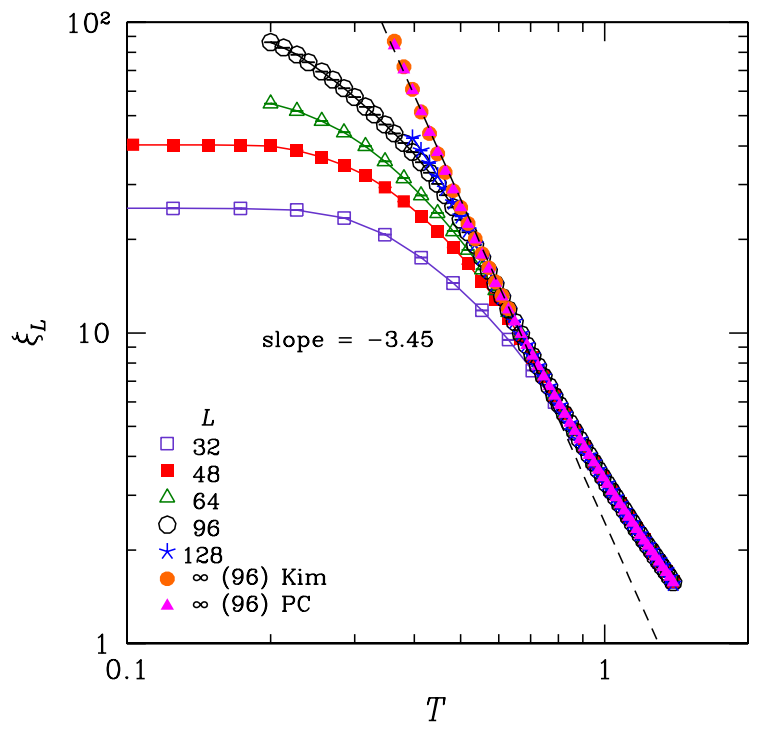

FIG. 6: (Color online) Log-log plot of the finite-size correlation length $\xi(L, T)$ as a function of temperature for several system sizes. The solid orange circles represent extrapolated data to the thermodynamic limit from $L=96$ using the method of $\mathrm{Kim}^{21}$ and the solid pink triangles represent the data extrapolated to $L=\infty$ using the method of Palassini and Caracciolo (PC) ${ }^{22}$ The (extrapolated) data seem to follow a power-law behavior with $\nu \approx 3.45$, which is close to the value of the critical exponent for Gaussian-distributed disorder, $\nu=3.54(2)$. The dashed line is a guide to the eye.

via differentiation of the data. For the available system sizes $\eta_{\text {eff }}(L, T)$ is always greater than $\sim 0.2$ (see Fig. 17). A reliable extrapolation to infinite $L$ and $T$ tending to zero would again require data of much larger system sizes.

There have been numerous estimates of $\eta$ at zero temperature for the two-dimensional Ising spin glass with bimodally distributed disorder, including direct measurements of the correlation function $G(r)$ by combinatorial or Monte Carlo methods,$\frac{10,12}{1}$ and there is a general consensus that $\eta \gtrsim 0.15$. Indeed it can be noted that the finite- $T$ raw $G(r)$ data in McMillan's ${ }^{12}$ Fig. 1 are by inspection incompatible with $\eta=0$.

From the definition of $\eta$ through $G(r)$, when there is a degenerate ground state at $T=0$, the time average spin-spin correlation function must decay with increasing distance $r$ implying a positive-definite value for $\eta$. A fortiori at any $T$ slightly above zero one would expect $G(r, T) \leq G(r, 0)$ for all $r$ except in quite exceptional cases. Otherwise, a limiting $\eta_{\mathrm{eff}}=0$ [meaning $G(r, T)=$ 1 for all $r$ at $T$ close to zero] appears to be ruled out from basic physical principles. 


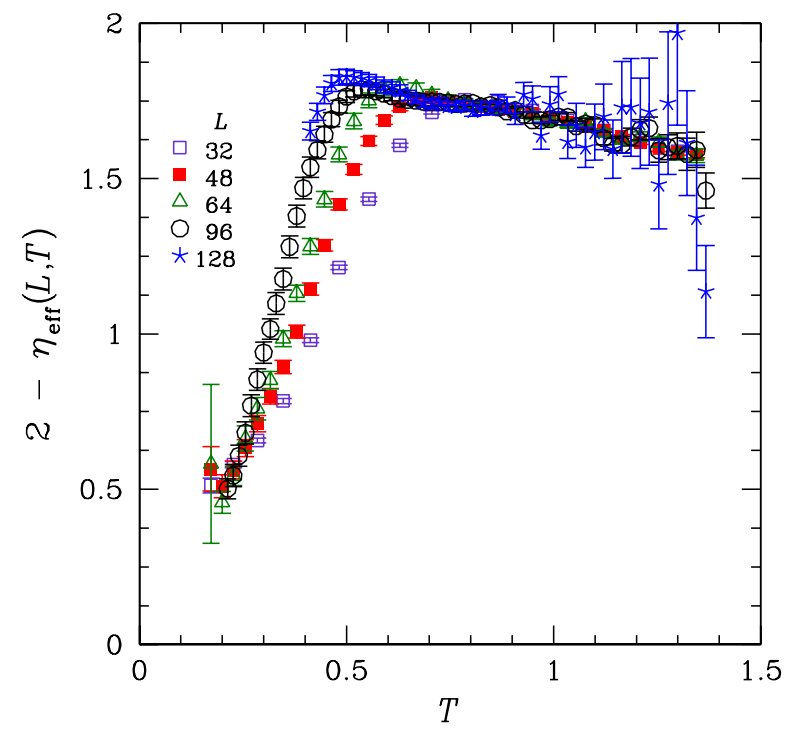

FIG. 7: (Color online) Effective exponent $2-\eta_{\text {eff }}(L, T)=$ $d \ln \left[\chi_{\mathrm{SG}}(L, T)\right] / d \ln [\xi(L, T)]$ as a function of temperature for different system sizes $L$. For all system sizes and temperatures studied $\eta_{\text {eff }}$ is always greater than 0.2 , although an extrapolation to $\eta_{\mathrm{eff}}=0$ cannot be ruled out.

\section{Gaussian disorder}

We have also computed the effective critical exponents for the correlation length $\left[\nu_{\text {eff }}(L, T)\right]$, the specific heat $\left[\alpha_{\text {eff }}(L, T)\right]$, the susceptibility $\left[\gamma_{\text {eff }}(L, T)\right]$, and correlation function $\left[\eta_{\mathrm{eff}}(L, T)\right]$ for the two-dimensional Ising spin glass with Gaussian-distributed disorder in order to test corrections to scaling in that model. In this case

$$
P\left(J_{i j}\right) \sim e^{-J_{i j}^{2} / 2 J}
$$

in Eq. (1). The results are qualitatively similar to the results found for the model with bimodally distributed disorder, but the data extend to lower temperatures thus making an extrapolation to zero temperature slightly more reliable. Still, without the knowledge of the zerotemperature estimate of the stiffness exponent $\theta, \nu=$ $-1 / \theta$ could not be determined to such high precision. In Fig. 8 we illustrate this behavior with data for $\eta_{\text {eff }}$ and $\nu_{\text {eff }}$ as a function of temperature. In Fig. 9 we compare the effective critical exponent $\gamma_{\text {eff }}^{-1}$ for Gaussian and bimodal disorder for $L=128$. While the extrapolation in the Gaussian case can be done easily to $T=0$, since the zero-temperature limit is well known, this is difficult for the bimodal case.



FIG. 8: (Color online) Effective exponent $2-\eta_{\mathrm{eff}}(L, T)=$ $d \ln \left[\chi_{\mathrm{SG}}(L, T)\right] / d \ln [\xi(L, T)]$ as a function of temperature for different system sizes $L$ for Gaussian disorder. The data extrapolate well to $\eta \approx 0$. Note that for $L=128$ the data for $T \gtrsim 0.9$ have been dropped due to strong fluctuations. Inset: Effective exponent $\nu_{\text {eff }}$ as a function of temperature for different system sizes $L$. The dashed line corresponds to the zero-temperature estimate from the stiffness exponent, $\nu=3.54(2)$.

\section{UNIVERSALITY AND FINITE-SIZE SCALING}

The critical values of the correlation length divided by the system size $\xi_{L}\left(T_{\mathrm{c}}\right) / L$ [Eq. (2)] and the Binder ratio $g\left(T_{\mathrm{c}}\right)[\mathrm{Eq}$. (8)] are characteristic of the universality class of a continuous transition. These are linked to $\eta$ at criticality because they represent various ratios of integrals with $G(r) \sim r^{d-2+\eta}$. For example, in a strip geometry for two space dimensions, $\xi_{L} / L=1 /(\pi \eta)$ at criticality ${ }^{36}$ The correlation length divided by system size of the twodimensional spin glass with Gaussian-distributed disorder diverges for $T \rightarrow 0$ and the Binder ratio tends to 1. If the two-dimensional Ising spin glass with bimodal interactions lies in the same universality class as the system with Gaussian disorder, then identical values for these parameters at criticality should be observed. The Binder ratio values $g(L, T)$ become temperature independent within the error bars for $T<T^{*}(L)$ providing estimates of the zero-temperature values $g(L, 0)$. There are corrections to scaling but the series of points appear to tend to a large- $L$ limit which is significantly less than unity.

For any continuous transition, at large $L$ and $T$ approaching $T_{\mathrm{c}}$ the Binder ratio is a nontrivial function of the variable $\xi_{L} / L, 27,37,38$ A plot of $g(L, T)$ vs $\xi_{L}(L, T) / L$ 


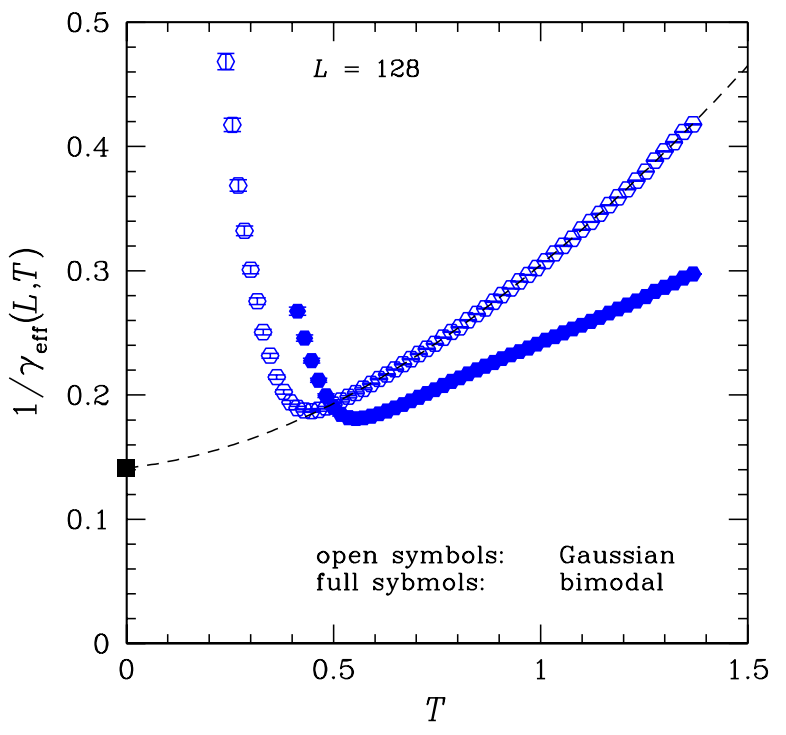

FIG. 9: (Color online) Effective critical exponent $1 / \gamma_{\text {eff for }}$ both Gaussian (open symbols) and bimodal disorder (full symbols) as a function of temperature $T$. While in the Gaussian case the $T=0$ limit is known $\left(\gamma^{-1} \approx 0.14\right.$, black square) and thus the extrapolation from the finite-temperature data can be performed via a simple third-order polynomial (dashed line), the data for the bimodal case can be extrapolated to any arbitrary value including $1 / \gamma_{\text {eff }}=0$, which corresponds to exponential scaling.

(Fig.10) shows a remarkable behavior: all points for both bimodal and Gaussian disorder are on a unique curve. This is particularly striking as the data span both the regions $T>T_{\xi}(L)$ and $T<T_{\xi}(L)$. There is, however, a qualitative difference between the Gaussian data and the bimodal data. For the former at each $L$ the data points extend to the same zero-temperature end point $\xi_{L}(L, T=0) / L=\infty$ and $g(L, T=0)=1$, while for the system with bimodal disorder the end points for different $L$ seem to cluster and not grow beyond $\xi_{L}(L, T=$ $0) / L \sim 0.91(2)$ and $g(L, T=0) \sim 0.92(2)$. In fact, the data do grow slightly, but the growth rate is within statistical error bars. This point can be interpreted as the zero-temperature critical parameters for this model. Thus, while the scaling functions agree, at zero temperature both models seem not to be in the same universality class. An alternative explanation could be that the model with bimodal disorder is "marginal," i.e., the endpoint might approach $g(L, T=0)=1$ logarithmically slow. In the bulk regime, which corresponds to the lower left corner of Fig. 10, data for $g(L, T)$ and $\xi_{L}(L, T) / L$ agree and thus suggest that both models might share a common finite-temperature universality class. Note that this cannot be inferred from studying the critical exponents due to large corrections to scaling, as shown in Sec. IV

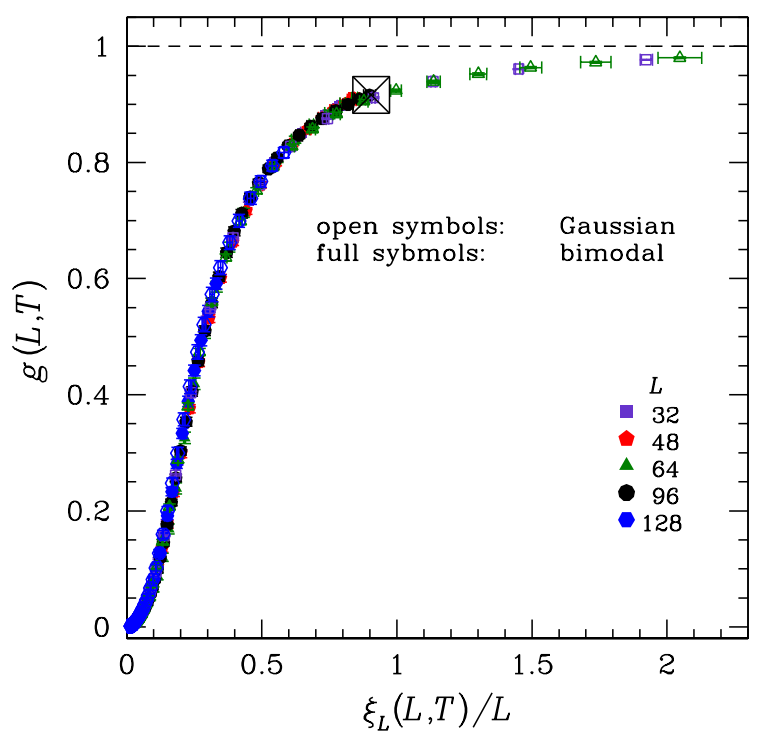

FIG. 10: (Color online) Binder ratio $g(L, T)$ as a function of the finite-size correlation length divided by the system size, $\xi_{L}(L, T) / L$. While the data for Gaussian disorder seem to extrapolate to $g(L, 0)=1$ and $\xi_{L}(L, T) / L=\infty$, the data for bimodal disorder seem to stop at $g(L, 0) \approx 0.92$ and $\xi_{L}(L, T) / L \approx 0.91$ (marked by a boxed cross).

\section{CONCLUSION}

We have carried out a careful numerical study of the two-dimensional Ising spin-glass model with bimodallydistributed disorder at finite temperatures with system sizes up to $L=128$, measuring the specific heat, finitesize correlation length, the spin-glass susceptibility, and the Binder ratio. The data neither confirm nor disprove the hypothesis 17 that in the large- $L$, low-but-nonzero- $T$ limit the effective critical exponents of the system are identical to the known critical exponents of the twodimensional Ising spin glass with Gaussian-distributed disorder, although the data seem to follow a power-law behavior at finite $T$ (see, for example, Fig. 11). Since the evidence for the critical exponents of the two-dimensional Ising spin glass with bimodally distributed disorder being identical to those of the pure Gaussian case is weak, a further critical analysis of the estimates for the other distributions studied in Ref. 38 might be opportune. A plot of the Binder ratio against the finite-size correlation length (Fig. 10), which shows very small corrections to scaling, only suggests that in the bulk regime and for finite temperatures both models might share a common universality class. Corrections to scaling are extremely large in the bimodal case. Therefore our main result is that simulations with larger system sizes and lower temperatures are needed to conclusively compute the critical exponents to prove the scenario proposed in Ref. 17 in which both models with Gaussian and bimodally dis- 
tributed disorder are in different universality classes at $T=0$, where the degeneracy of the ground state plays a key role, yet the models share the same universality class at finite nonzero temperatures and very large system sizes. ful discussions, and T. Jörg for pointing out the usefulness of plotting the data in the way shown in Fig. 10. The simulations were performed on the Hreidar and Gonzales clusters at ETH Zürich.

\section{Acknowledgments}

We would like to thank R. Fisch, K. Hukushima, T. Jörg, J. Poulter, M. Troyer, and A. P. Young for fruit-

${ }^{1}$ K. Binder and A. P. Young, Spin glasses: Experimental facts, theoretical concepts and open questions, Rev. Mod. Phys. 58, 801 (1986).

2 J.-S. Wang and R. H. Swendsen, Low-temperature properties of the $\pm J$ spin glass in two dimensions, Phys. Rev. B 38, 4840 (1988).

${ }^{3}$ L. Saul and M. Kardar, Exact integer algorithm for the twodimensional $\pm J$ Ising spin glass, Phys. Rev. E 48, R3221 (1993).

4 J. Houdayer, A cluster Monte Carlo algorithm for 2dimensional spin glasses, Eur. Phys. J. B. 22, 479 (2001).

5 J. Lukic, A. Galluccio, E. Marinari, O. C. Martin, and G. Rinaldi, Critical thermodynamics of the two dimensional $\pm J$ Ising spin glass, Phys. Rev. Lett. 92, 117202 (2004).

${ }^{6}$ H. G. Katzgraber and L. W. Lee, Correlation length of the two-dimensional Ising spin glass with bimodal interactions, Phys. Rev. B 71, 134404 (2005).

7 S. F. Edwards and P. W. Anderson, Theory of spin glasses, J. Phys. F: Met. Phys. 5, 965 (1975).

8 A. K. Hartmann and A. P. Young, Lower critical dimension of Ising spin glasses, Phys. Rev. B 64, 180404(R) (2001).

9 H. Rieger, L. Santen, U. Blasum, M. Diehl, M. Jünger, and G. Rinaldi, The critical exponents of the two-dimensional Ising spin glass revisited: exact ground-state calculations and Monte Carlo simulations, J. Phys. A 29, 3939 (1996).

10 J. A. Blackman, J. R. Gonçalves, and J. Poulter, Properties of the two-dimensional random-bond +/-J Ising spin glass, Phys. Rev. E 58, 1502 (1998).

11 J. Poulter and J. A. Blackman, An exact algorithm for spin correlation functions of the two dimensional $+/-J$ Ising spin glass in the ground state, Phys. Rev. B 72, 104422 (2005).

12 W. L. McMillan, Monte Carlo simulation of the twodimensional random (+/-J) Ising model, Phys. Rev. B 28, 5216 (1983).

13 R. N. Bhatt and A. P. Young, Numerical studies of Ising spin glasses in two, three and four dimensions, Phys. Rev. B 37, 5606 (1988).

14 C. Amoruso, A. K. Hartmann, M. B. Hastings, and M. A. Moore, Conformal Invariance and SLE in Two-Dimensional Ising Spin Glasses (2006), (condmat/0601711).

15 J.-S. Wang, Worm algorithm for two-dimensional spin glasses, Phys. Rev. E 72, 036706 (2005).

16 R. Fisch, Sub-extensive singularity in the $2 D \pm J$ Ising spin glass (2006), (cond-mat/0607622).
17 T. Jörg, J. Lukic, E. Marinari, and O. C. Martin, Strong Universality and Algebraic Scaling in Two-Dimensional Ising Spin Glasses, Phys. Rev. Lett. 96, 237205 (2006).

18 K. Hukushima and K. Nemoto, Exchange Monte Carlo method and application to spin glass simulations, J. Phys. Soc. Jpn. 65, 1604 (1996).

19 E. Marinari, Optimized Monte Carlo methods, in Advances in Computer Simulation, edited by J. Kertész and I. Kondor (Springer-Verlag, Berlin, 1998), p. 50, (condmat/9612010).

20 F. Cooper, B. Freedman, and D. Preston, Solving $\phi_{1,2}^{4}$ theory with Monte Carlo, Nucl. Phys. B 210, 210 (1982).

21 J. K. Kim, Asymptotic scaling of the mass gap in the twodimensional $O(3)$ nonlinear sigma model: A numerical study, Phys. Rev. D 50, 4663 (1994).

22 M. Palassini and S. Caracciolo, Universal Finite-Size Scaling Functions in the 3D Ising Spin Glass, Phys. Rev. Lett. 82, 5128 (1999).

${ }^{23}$ H. G. Ballesteros, A. Cruz, L. A. Fernandez, V. MartinMayor, J. Pech, J. J. Ruiz-Lorenzo, A. Tarancon, P. Tellez, C. L. Ullod, and C. Ungil, Critical behavior of the threedimensional Ising spin glass, Phys. Rev. B 62, 14237 (2000).

24 L. W. Lee and A. P. Young, Single spin- and chiral-glass transition in vector spin glasses in three dimensions, Phys. Rev. Lett. 90, 227203 (2003).

${ }^{25}$ H. G. Katzgraber, L. W. Lee, and A. P. Young, Correlation length of the two-dimensional Ising spin glass with Gaussian interactions, Phys. Rev. B 70, 014417 (2004).

${ }^{26}$ K. Binder, Critical properties from Monte Carlo coarse graining and renormalization, Phys. Rev. Lett. 47, 693 (1981).

27 H. G. Katzgraber, M. Körner, and A. P. Young, Universality in three-dimensional Ising spin glasses: A Monte Carlo study, Phys. Rev. B 73, 224432 (2006).

28 Note that the specific heat can also be computed as a derivative of the internal energy $E=-[\langle\mathcal{H}\rangle]_{\mathrm{av}}$, i.e., $C_{V}=$ $\partial E / \partial T$ (constant volume). We have also computed the specific heat using this method and the results agree perfectly with the results using Eq. (9).

29 I. A. Campbell, A. K. Hartmann, and H. G. Katzgraber, Energy size effects of two-dimensional Ising spin glasses, Phys. Rev. B 70, 054429 (2004).

${ }^{30}$ G. A. Baker, Jr. and J. C. Bonner, Scaling behavior at zerotemperature critical points, Phys. Rev. B 12, 3741 (1975).

31 Numerically one can only access finite system sizes. In this case the boundary conditions (here periodic) as well as the 
shape anisotropy of the models compared have to be the same. Only then will the models be in the same universality class, provided that the critical exponents as well as the values of different observables at criticality are the same.

32 Similar results have been found via an alternate analysis in Ref. 39, although it is unclear if an extrapolation from the high-temperature behavior to $T=0$ is plausible.

${ }^{33}$ Note that Jörg et al. in Ref. 17 also study other disorder distributions, such as the gap 1/4 case, where corrections to scaling are much smaller than for bimodally distributed disorder. For these models, the data seem compatible with the critical behavior of the model with Gaussian disorder.

34 B. Efron and R. J. Tibshirani, An Introduction to the Bootstrap (Chapman \& Hall, 1994).

35 S. Caracciolo, R. G. Edwards, S. J. Ferreira, A. Pelissetto, and A. D. Sokal, Extrapolating Monte Carlo Simulations to Infinite Volume: Finite-Size Scaling at $\xi / L \gg 1$, Phys. Rev. Lett 74, 2969 (1995).

36 J. L. Cardy, Finite-size scaling in strips: antiperiodic boundary conditions, J. Phys. A 17, L961 (1984).

37 J.-K. Kim, A. J. F. de Souza, and D. P. Landau, Numerical computation of finite size scaling functions: An alternative approach to finite size scaling, Phys. Rev. E 54, 2291 (1996).

38 T. Jörg, Critical behavior of the three-dimensional bonddiluted Ising spin glass: Finite-size scaling functions and universality, Phys. Rev. B 73, 224431 (2006).

39 H. G. Katzgraber, L. W. Lee, and I. A. Campbell, Nontrivial critical behavior of the free energy in the twodimensional Ising spin glass with bimodal interactions (2005), (cond-mat/0510668). 